\title{
Supporting Information: Gigahertz nano-optomechanical resonances in a dielectric SiC-membrane metasurface array
}

Idris A. Ajia ${ }^{1 *}$, Jun-Yu Ou ${ }^{2}$, Nicholas J. Dinsdale ${ }^{1}$, Haobijam Johnson Singh ${ }^{1}$, Theo Chen-Sverre ${ }^{1}$, Tongjun Liu ${ }^{2}$, Nikolay I. Zheludev ${ }^{2,3}$, and Otto L. Muskens ${ }^{1 *}$

${ }^{1}$ Physics and Astronomy, Faculty of Physical Sciences and Engineering, University of Southampton, SO17 1BJ, Southampton, UK

${ }^{2}$ Optoelectronics Research Centre and Centre for Photonic Metamaterials, University of Southampton, Southampton, SO17 1BJ, United Kingdom

${ }^{3}$ Centre for Disruptive Photonic Technologies, School of Physical and Mathematical Sciences and The Photonics Institute, Nanyang Technological University, Singapore 637378, Singapore

Corresponding authors: omuskens@soton.ac.uk, idris.ajia@soton.ac.uk

S1. Determining the effects of ambient pressure on the modes

(a)

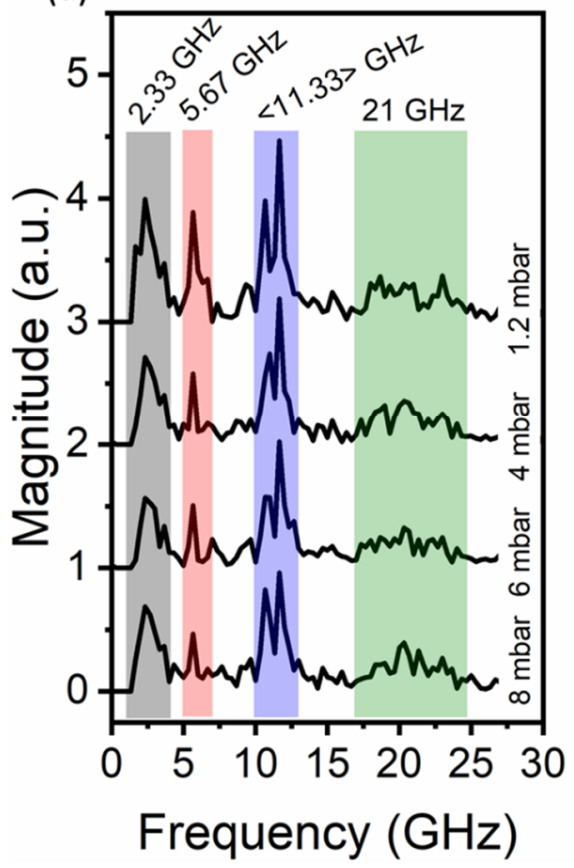

(b)

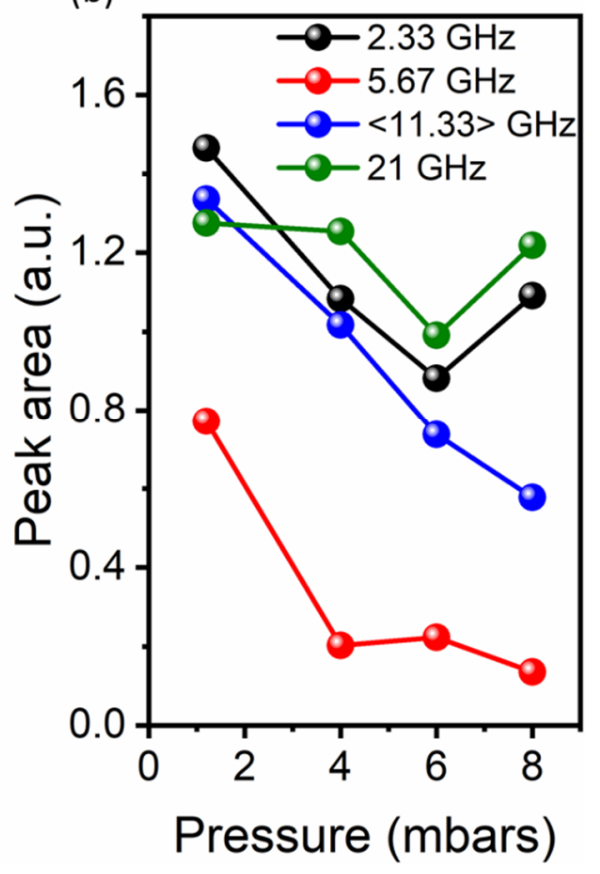

Fig. S 1 (a) Stacked FFT of pressure dependent measurements showing the individual eigenmodes of the MSA. (b) Pressure dependent response of the resonance peak area of the respective modes.

To shed light on the effect of dampening on the optomechanical response of the MSA, pump-probe measurements were carried out at different pressures in the vacuum cell holding the MSA. Fig $S 1$ (a) Shows the stacked pressure dependent FFT spectra of $\triangle R / R$. We have treated each of the low (2.33 $\mathrm{GHz})$, intermediate $(5.67 \mathrm{GHz})$ and high $(<11.33>\mathrm{GHz}$ set of modes) gigahertz regions as independent mechanical oscillators and plotted their integrated magnitude against pressure in Fig. $S 1$ (b). We observe a decline in the magnitude of the optomechanical modes $(2.33 \mathrm{GHz}, 5.67 \mathrm{GHz}$ and $<11.33>$ 
$\mathrm{GHz}$ ) with increasing pressure inside the vacuum cell. The $21 \mathrm{GHz}$ mode is less susceptible to pressure change than the other modes. That said, it is also very noisy due to its low intensity. This may have dampened its pressure response. It is also quite possibly related to due to Brillouin scattering within the SiC membrane, ${ }^{1,2}$ which should make it less responsive to ambient pressure than the optomechanical modes.

\section{S2. Multi-damped oscillator model}

A multi-damped oscillator model was used to extract the principal mechanical modes from the timedomain data of $\Delta T / T$ and $\Delta R / R$ in three separate spectral bands. Fourier bandpass filters were used to select frequency ranges $1.5 \leq \mathrm{f} \leq 3 \mathrm{GHz}, 4 \leq \mathrm{f} \leq 8.5 \mathrm{GHz}$ and $\mathrm{f} \geq 10 \mathrm{GHz}$ were used for the low, intermediate and high gigahertz modes respectively. In each band, the three main components were obtained using a multimodal fit with three damped oscillator terms according to

$\frac{\Delta T}{T}(t)=y_{0}+A_{1} e^{-t / \tau_{1}}\left[\sin \left(2 \pi \frac{t-t_{c 1}}{T_{1}}\right)\right]+A_{2} e^{-t / \tau_{2}}\left[\sin \left(2 \pi \frac{t-t_{c 2}}{T_{2}}\right)\right]+A_{3} e^{-t / \tau_{3}}\left[\sin \left(2 \pi \frac{t-t_{c 3}}{T_{3}}\right)\right]$.

Here $A_{1}, A_{2}$ and $A_{3}$ represent the maximum amplitudes of each of the oscillators, $\tau_{1}, \tau_{2}$ and $\tau_{3}$ represent the decay constants of the damping term, $t$ is the independent time variable, $t_{1}, t_{2}$ and $t_{3}$ are the phase components and $T_{1}, T_{2}$ and $T_{3}$ are the periods of each of the oscillators.

Results for the time response results in three spectral bands are shown in Fig. $S 2$ (a), (b) and (c) for transmission data $\Delta T / T$ and (e), (f) and (g) for reflection data $\Delta R / R$, respectively. Fig. $S 2$ (d) and (h) show the sum of the fitted multi-damped oscillators superimposed on the experimental $\Delta T / T$ and $\Delta R / R$ responses of the MSA, respectively. Overall, the simplified model of 9 distinct damped oscillators captures well most of the observed response, as demonstrated by the low values of the residuals shown in Fig. S 2 (d) and (h) (vertically offset for clarity). 
(a) $\times 10^{-5}$

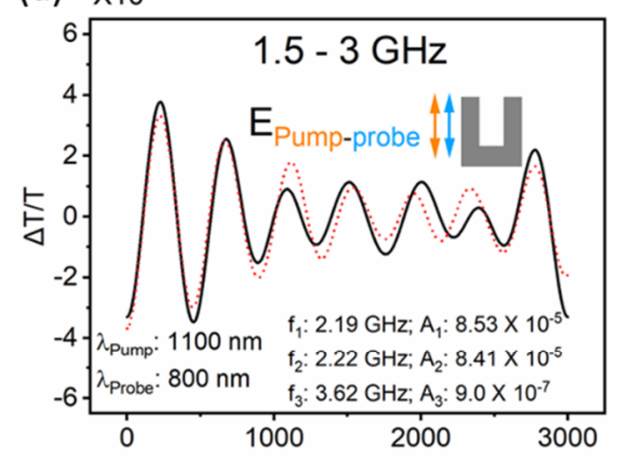

(c)

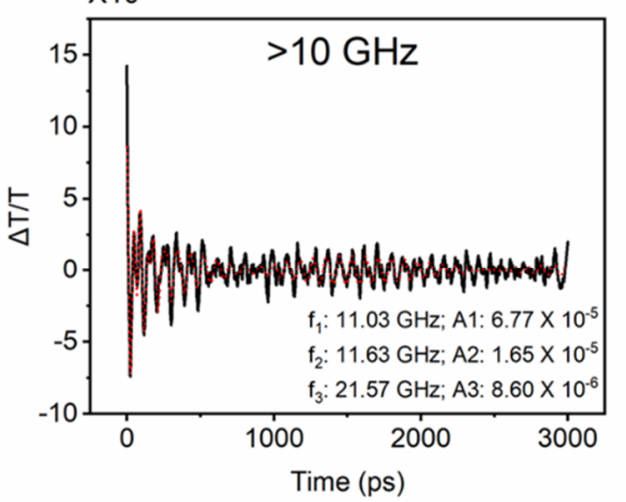

(e) $\times 10^{-6}$
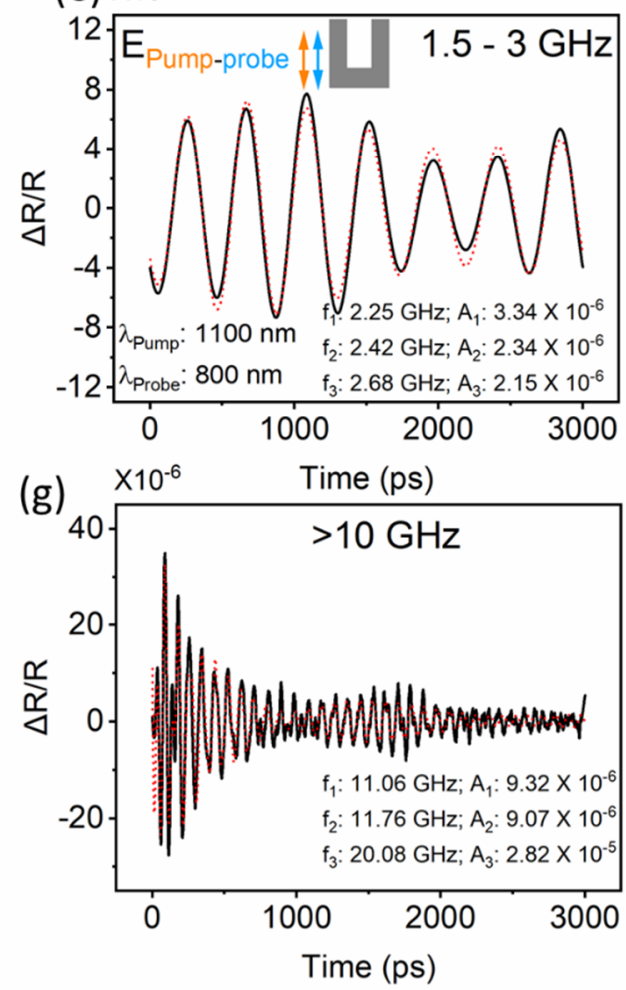

Sum of residuals

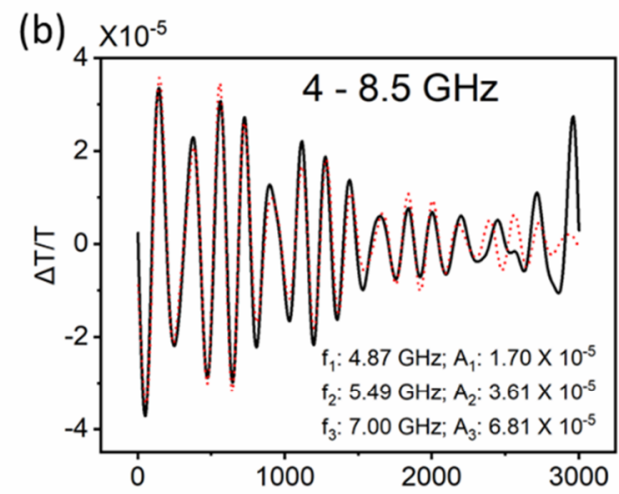

(d)

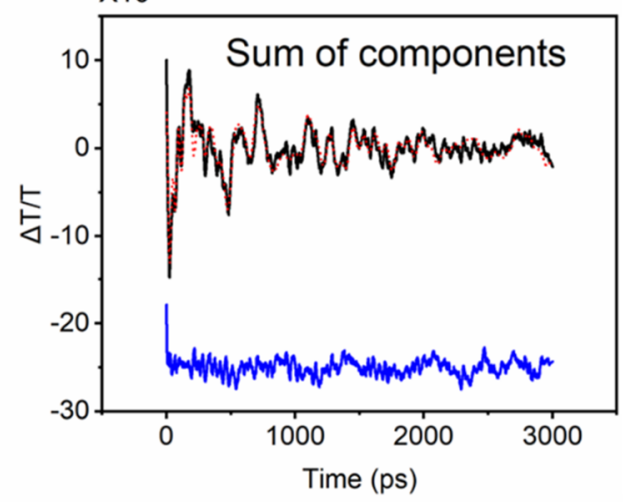

(f) $\times 10^{-6}$
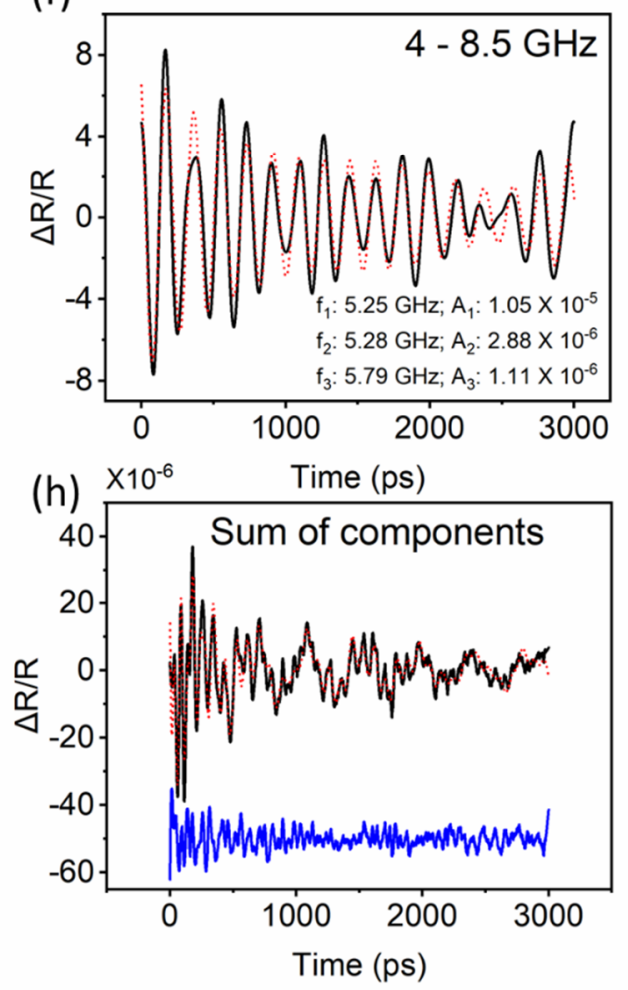

Fig. S 2: FFT filtered signals (black solid lines) and multi-damped oscillator fit results (red dashed lines) of the individual modes for ranges $(\Delta T / T, \Delta R / R)$ : $(a, e) 1.5 \leq f \leq 3 \mathrm{GHz},(b, f) 4 \leq f \leq 8.5 \mathrm{GHz},(c, g) f>10$ $\mathrm{GHz}$ and $(\mathrm{d}, \mathrm{h})$ sum of multi-damped oscillator fits superimposed on the time-domain experimental data, with total residuals between fitted model and experiment (blue sold line) 


\section{S3. Probe signal analysis: dependence on wavelength}

We carried out probe wavelength dependent measurements on the MSA while keeping the pump wavelength at $1100 \mathrm{~nm}$. The probe wavelength dependent measurements required changing the primary beam from the Chameleon Ti:Sa laser, which necessitated a retuning of the optical parametric oscillator (OPO) for every probe wavelength step. This introduced an additional layer of complexity, that is not experienced with tuning of the pump wavelength, which may have resulted in slight alterations in the spatial and temporal overlap of the pump and probe beams relative to each other. As such, we observe slight variations in the frequencies of the vibrational modes. The time varying signals are shown in the left panels of Fig. $S 3$ (a) for $\Delta R / R$ and (c) $\Delta T / T$ signal and the corresponding fast Fourier transform signals are shown in the right panels of Fig. $S 3$ (a) and (c).

To extract the magnitude of the individual modes at different probe wavelengths, the modes were integrated over the shaded regions in the in the right panels of Fig. S 3 (a) and (c). The wavelength dependence of the modes is presented in Fig. $S 3$ (b) and (d), corresponding to the y-axis on the right side of the plot. The angled bracket notations are used to indicate a range of frequencies around the ones specified within the brackets.

Since we are primarily concerned with the coupling of the modes to the optical transmission and reflection signals, the wavelength dependences of the modes are superimposed on the transmission and reflection spectra of the MSA around the tuning range. It is observed that the peaks in the signal strength correspond to the positions where the slopes in the spectral response are the steepest. We therefore attribute the sensitivity of the vibrational signals to the probe wavelength to the increased sensitivity of the spectrum at the steep edges of the features in $R$ and $T$.

As the modes are most responsive to the probe wavelength of $800 \mathrm{~nm}$, we have chosen to fix the probe wavelength at $800 \mathrm{~nm}$ for all subsequent experiments, unless otherwise stated. To check the effect of the probe signal on the vibrational modes, the beam paths of the pump and probe signals are temporarily swapped, so that $800 \mathrm{~nm}$ becomes the pump beam and $1100 \mathrm{~nm}$ is the probe beam. The result of this test, shown in Fig. 54 , reveals no significant effect. 
(a) $\times 10^{-5}$

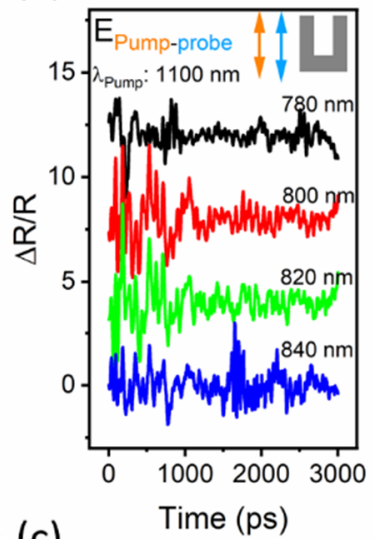

(c)

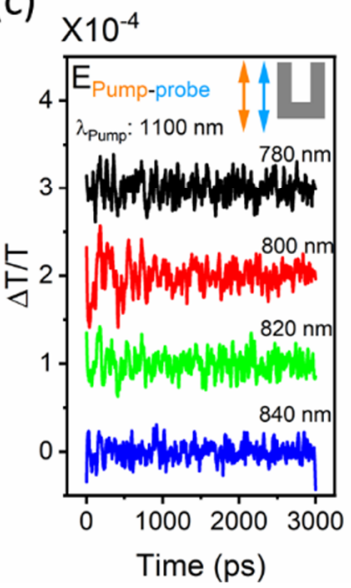

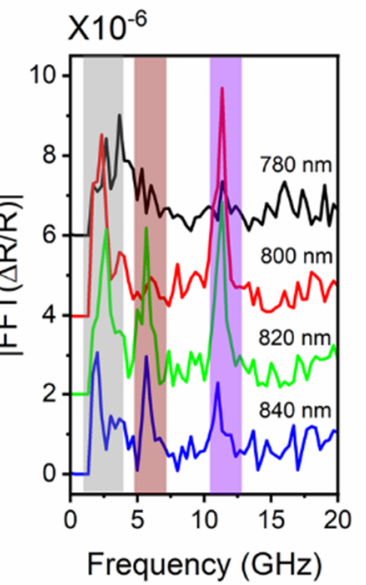

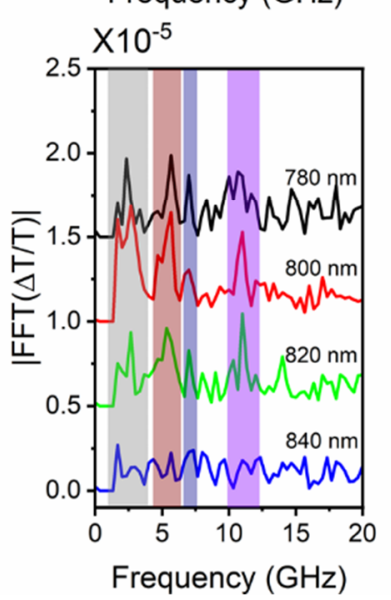

(b)

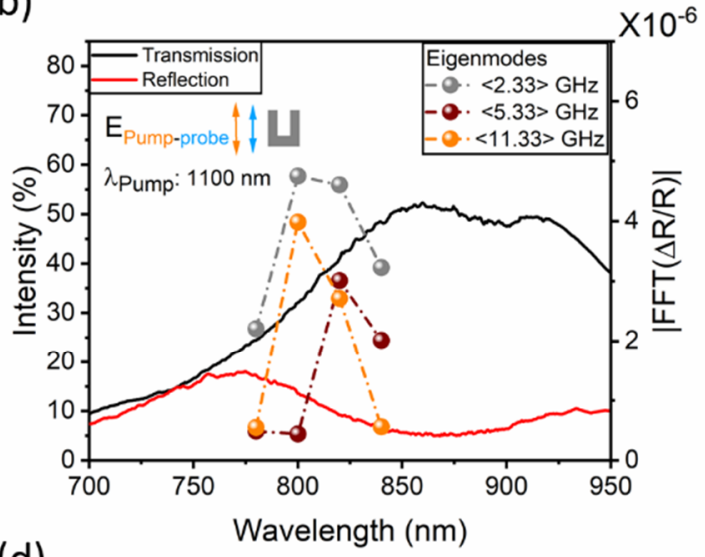

(d)

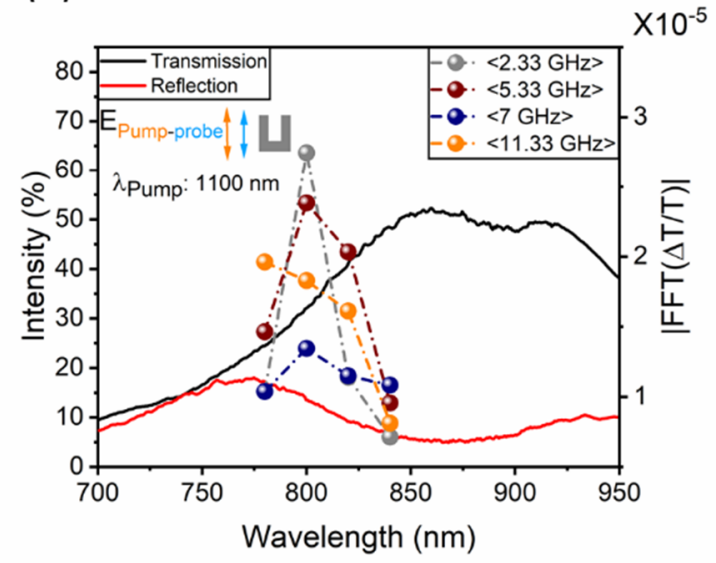

Fig. $S 3(a, d) \Delta R / R$ and $\Delta T / T$ response of the probe wavelength dependence of the nanocantilevers in time domain (left panel) and frequency domain (right panel). (Stacked for clarity) (b, d) The wavelength dependence of the vibrational modes amplitudes superimposed on the transmission and reflection experimental spectra.
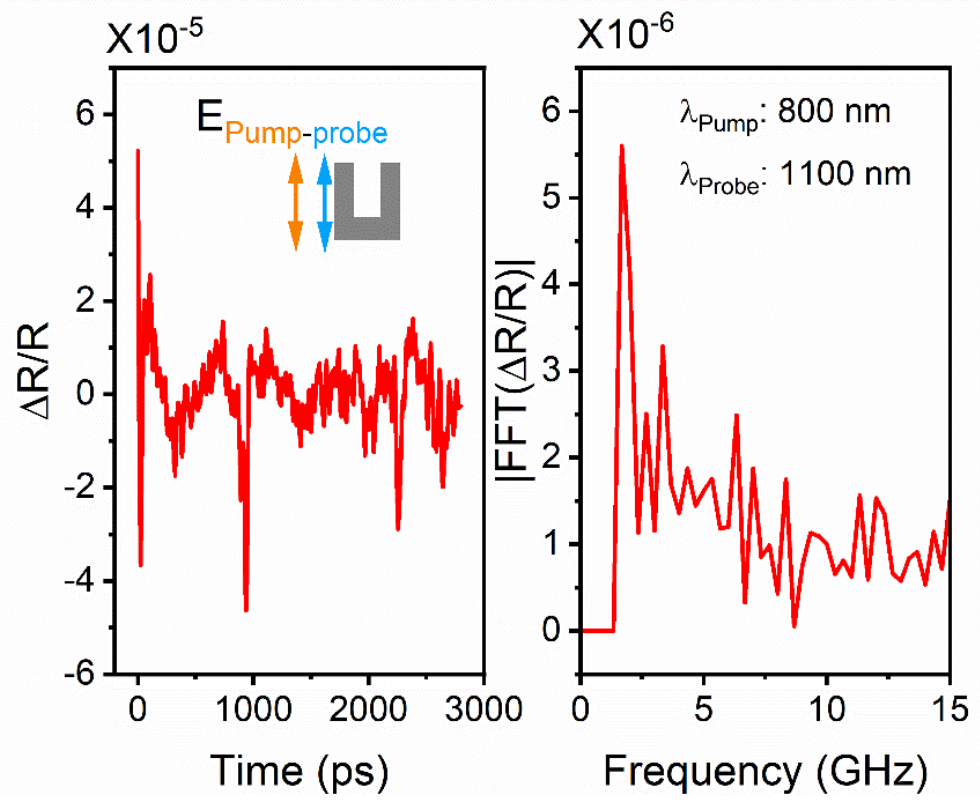

Fig. $S 4 \Delta R / R$ response of the nanocantilevers in time domain (left panel) and frequency domain (right panel) when the MSA was pumped at $800 \mathrm{~nm}$ and probed at $1100 \mathrm{~nm}$. 


\section{S4. COMSOL Mechanical Simulation Models}

COMSOL Multiphysics software was used to simulate the mechanical modes of the cantilever as well as the supporting structures of the nanocantilever, using a single metamolecule model and a $5 \times 5$ model based on the SiC MSA structure, respectively. The structural properties of SiC were acquired from existing literature and are shown in Table S. 1. ${ }^{3,4}$

\begin{tabular}{|l|r|l|}
\hline SiC material properties & Values & Reference \\
\hline Young's modulus (GPa) & 340 & 3 \\
\hline Density $\left(\mathrm{kg} \cdot \mathrm{m}^{-3}\right)$ & 3216 & 4 \\
\hline Refractive index & $\begin{array}{l}\text { Interpolation curve } \\
\text { from existing } \\
\text { literature. }\end{array}$ & 5 \\
\hline
\end{tabular}

Table S. 1: Structural properties of SiC used in simulation.

The single metamolecule is a $300 \mathrm{~nm}$ thick $655 \mathrm{~nm} \times 565 \mathrm{~nm}$ membrane as shown in Fig. S 5 (a). The dimensions as well as region where the optical load was applied is shown in the same figure. For the calculation, both Eigenfrequency and frequency domain solvers were used to extract specific Eigenfrequencies as well as the full frequency spectrum of the mechanical modes, respectively. The Eigenfrequency solver has the advantage of providing, in discrete form, the exact frequencies of the expressed mode, whereas the frequency domain solver returns a frequency domain representation of the vibrational response of the structure. However, a compromise is to be made between interval precision and solving time, which may occasionally lead to certain modes being omitted. To extract the volumetric and component displacement results, the calculation was limited to the surface integral of the top surface and the side boundaries of the nanocantilever in the metamolecule. This was sufficient to capture volumetric, $U_{x}, U_{y}$ and $U_{z}$ displacements.

In order to calculate the mechanical modes of the support structures, the boundaries surrounding the cantilever will have to be free (unfixed). However, COMSOL's frequency domain calculations require fixed reference points. As such, we created a $5 \times 5$ model with fixed external boundaries, but free internal support structures. This time, the optical load is applied on a $3 \times 3$ array of metamolecules, as shown in Fig. $S 5$ (b). To extract the modes that pertain only to the support structure, the results of the frequency domain solver was extracted from the indicated surface in Fig. 55 (b). The model in Fig. $\mathrm{S} 5$ (c) is the vibrational response at $5.33 \mathrm{GHz}$, which demonstrates that this mode is mostly from the support structure of the enclosing the nanocantilever. By extracting our results from only the supporting structures of the $5 \times 5$ array, we assume that any coupling between adjacent nanocantilevers is not significant enough to affect our detection of the modes from the support structure. This assumption is justified because only a small number $(4-9)$ of metamolecules are pumped and probed at any given time. The localization of the vibrational response on the support structures in Fig. $S 5$ (c) is consistent with our assumption at $5.33 \mathrm{GHz}$. The support structures also shows some high intensity delocalized modes below $1.5 \mathrm{GHz}$. These modes have been truncated from the presented result for clarity and because they are beyond the detection range of our current experimental setup. 


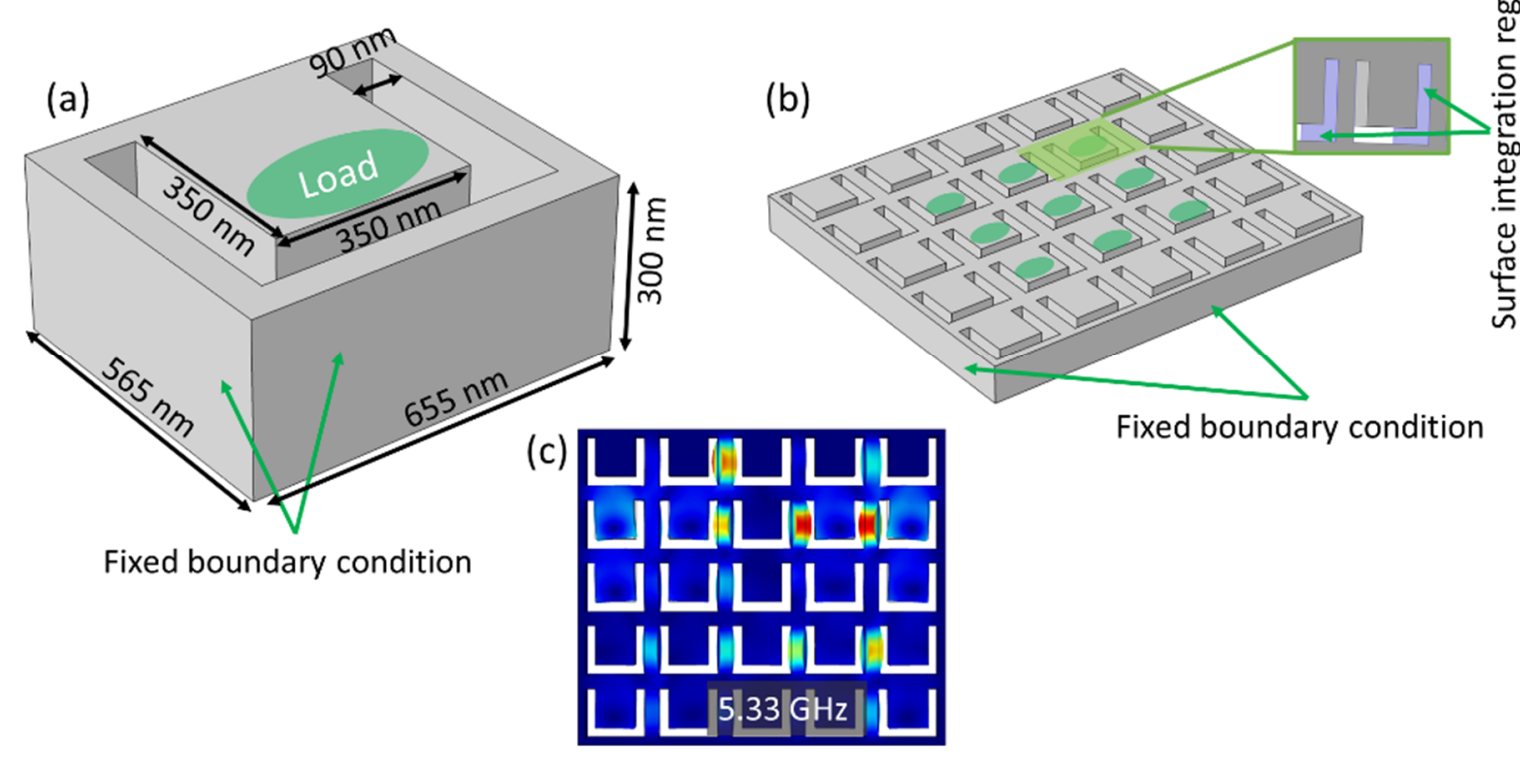

Fig. S 5 (a) Single metamolecule model showing the dimensions. (b) A $5 \times 5$ model of the metasurface array structure. (inset): Highlighted region showing the integrated surface area from where the support structure modes are extracted. (c) A full $5 \times 5$ model showing the vibrational mode of the support structure at $5.33 \mathrm{GHz}$.

\section{S5. Comparison between Si and SiC materials}

In this section, the mechanical response of Si metamolecule of the same dimensions as the SiC structure is simulated using the same method previously described, which is of interest for comparing with previous work. At this point we have not prepared similar Si structures for a direct experimental comparison. In this simulation, the Young's modulus of the Si membrane is set at $190 \mathrm{GPa}$, and the density is set at $2329 \mathrm{Kg} \mathrm{m}^{-3} .6,7$ The Responses of Si and SiC are plotted in Fig. S 6 (a) for comparison. We observe that virbrational modes of the nanocantilever of the Si metamolecule are similar to that of the SiC, but only slightly shifted to lower frequencies due to its lower Young's modulus. The main vibrational mode occurs at $1.9 \mathrm{GHz}$, with the other modes occurring at $5.3 \mathrm{GHz}, 5.7 \mathrm{GHz}, 10.1 \mathrm{GHz}$, $10.5 \mathrm{GHz}, 11.4 \mathrm{GHz}$, and $13.7 \mathrm{GHz}$.

We also ran an optical simulation that follows the previously described method. The interpolation curve of the refractive index is gotten from literature. ${ }^{8}$ The result is shown in Fig. $S 6$ (b). The absorption curve has several resonances with peaks at 850, $1200 \mathrm{~nm}$ and $1330 \mathrm{~nm}$. The higher refractive index of Si compared to $\mathrm{SiC}$ means that the spectrum of optical modes are correspondingly shifted to longer wavelengths.. This suggests that, while mechanical modes depend less on the material, the optical resonances of the driving and detecting beams will be more tunable by the choice of suitable dielectrics materials. The independent tunability of mechanical and optical characteristics and demonstrates the flexibility that these dielectric materials may offer for applications. 
(a)

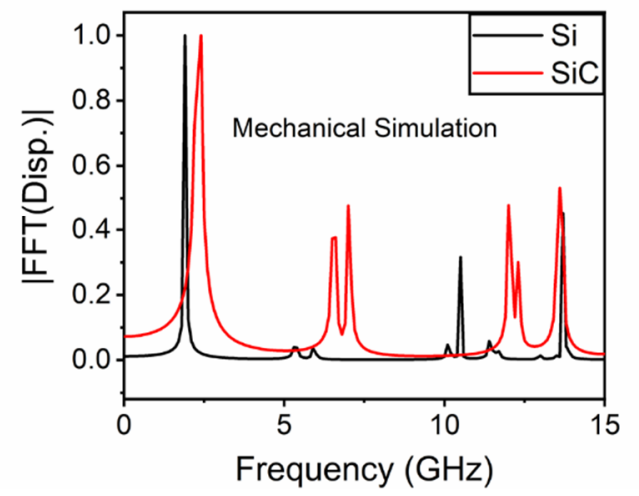

(b)

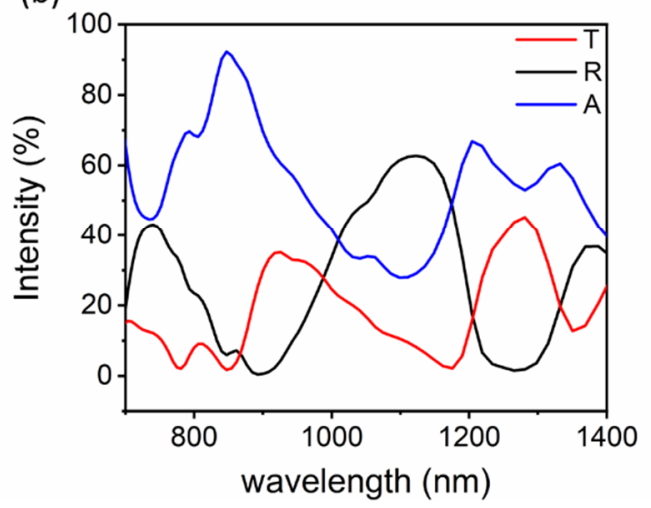

Fig. S 6 (a) Simulated mechanical modes of Si and SiC metamolecules. (b) Simulated optical response of a Si MSA showing transmission, reflection and absorption spectra.

\section{S6. $7 \mathrm{GHz}$ mode analysis}

Fig. S 7 (a) and (b) show the pump wavelength dependence of $\Delta T / T$ and $\Delta R / R$, respectively, in time and frequency domain. Although all experimentally observed modes are coupled to the $\Delta T / T$ and $\Delta R / R$ signals, there are differences in the resolutions and coupling strengths of each of the modes between $\Delta T / T$ and $\Delta R / R$. In general, $\Delta R / R$ appears less sensitive to the weaker intermediate modes, which results in the main modes at $2.33,5.33,11$ and $11.67 \mathrm{GHz}$ being more highly resolved. On the other hand, $\Delta T / T$ appears more sensitive to intermediate modes, which makes the main modes less resolved. The $7 \mathrm{GHz}$ mode (highlighted with red-shaded region in the frequency domain plots) is one of such intermediate modes which couple more strongly to $\Delta T / T$ than $\Delta R / R$.

In Fig. S 8, the side-by-side comparison of the aerial dependent measurements in frequency domain for $\Delta T / T$ and $\Delta R / R$ are shown for a range of positions on the MSA as indicated in Fig. $S 8$ (a). It is important to again highlight that both transmission and reflection response were measured simultaneously on exactly the same locations. Therefore, the variations between $\Delta T / T$ and $\Delta R / R$ represent local fluctuations in the coupling strength to different modes. Overall, both follow similar trends in terms of the small variations in mode frequency.

(a)

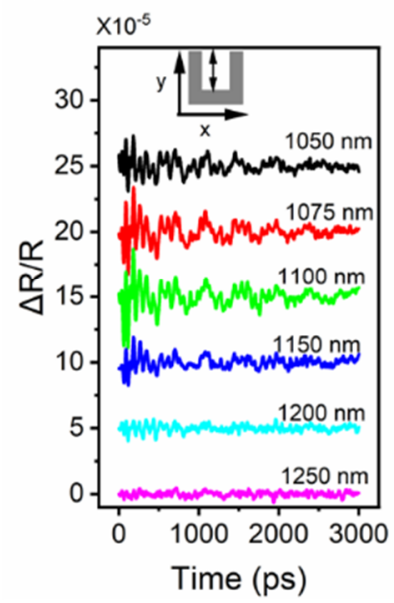

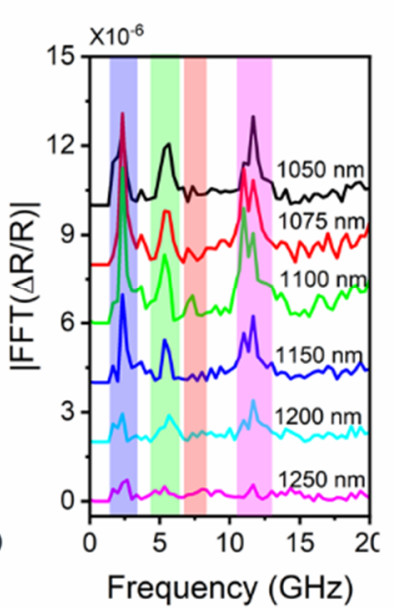

(b)

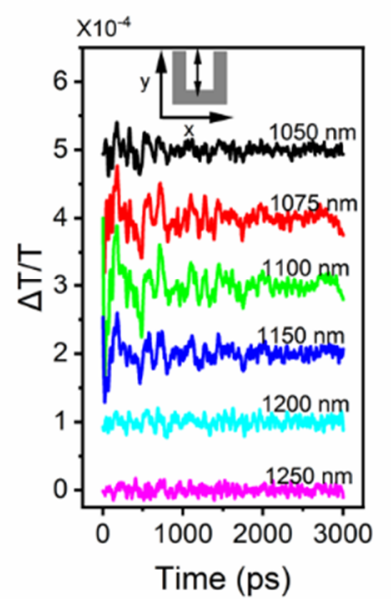

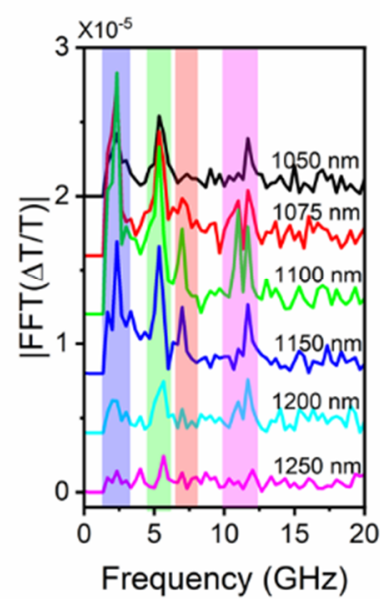

Fig. S 7(a) $\Delta T / T$ response of the pump wavelength dependence of the nanocantilevers in time domain (left panel) and frequency domain (right panel). (b) $\Delta R / R$ pump wavelength dependence of the nanocantilevers in time domain (left panel) and frequency domain (right panel). Measurements are vertically stacked for clarity. 
(a)

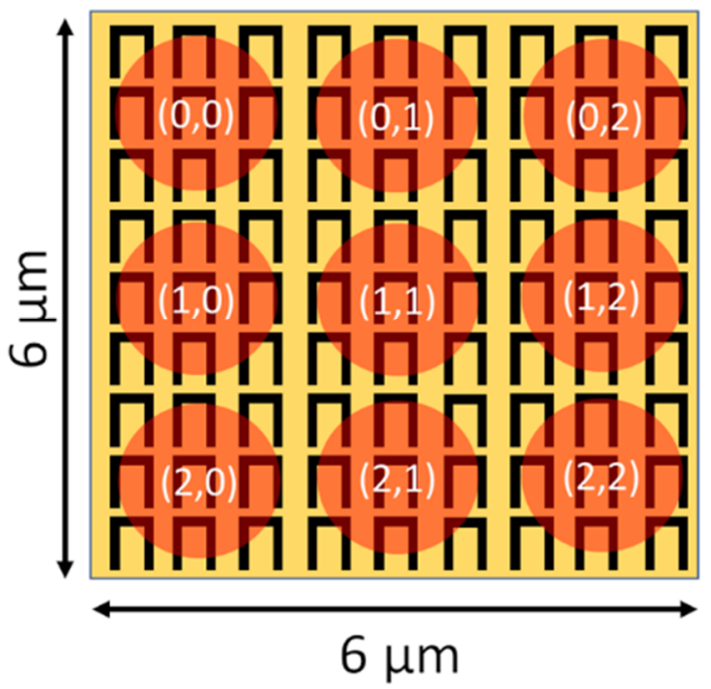

(b)

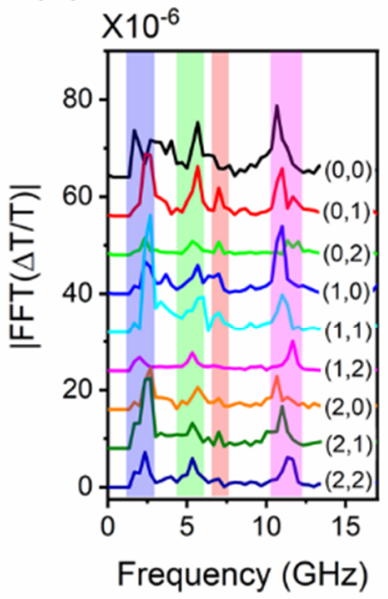

Fig. S 8 (a) Illustration of the aerial pump-probe scan of the MSA with labelled scan regions. (b) aerial dependence of the vibrational modes in frequency domain for $\Delta T / T$ (left panel) and $\Delta R / R$ (right panel). Measurements in (b) are vertically stacked for clarity.

\section{S7. Polarization dependent hyperspectral maps of the membrane}

To better visualize the local variations in the MSA response, porlarization dependent hyperspectral transmittance experiments were carried out on a $5 \mu \mathrm{m} \times 5 \mu \mathrm{m}$ area on the MSA. The resulting average transmittance spectra are shown in Fig. $S 9$ (a) for vertical $\left(E_{y}\right)$ and horizontal $\left(E_{x}\right)$ beams. Much of the polarization effect on optical transmittance occurs below $900 \mathrm{~nm}$. The hyperspectral images for the shaded regions, representing the probe range $(780 \mathrm{~nm}-840 \mathrm{~nm})$ and the pump range $(1050 \mathrm{~nm}-$ $1250 \mathrm{~nm}$ ) are shown in Fig. $S 9$ (b). The top panels show the dependence of the probe beam range on polarization, whereas the polarization dependence of the pump beam range is shown in the bottom panels of Fig. $S 9$ (b). We observe that the polarization dependence is more prominent in the probe wavelength range. We also find that there is some variation of the spectral response of the metasurface over the membrane area, linking to results shown in Figure $S 8$ which show variations in the mechanical vibrational spectra as well as its coupling to the optical pump and probe.

In addition, to understand theoretically how the MSA responds to horizontally polarized pumping, we ran numerical simulations, setting the polarization of the incident optical pump radiation to the horizontal direction. The near-field distribution maps Fig. S 10 (a)) of the horizontally polarized electric field is extracted at $1200 \mathrm{~nm}$. The XY plane of the nearfield distribution was acquired from of depth of $100 \mathrm{~nm}$ inside the structure. The corresponding field distribution along the $\mathrm{YZ}$ plane are also shown in the figure. The dashed lines indicate approximate location from which the planes were cut in the figures. The experimental spectrum in Figure 1 in the main text shows similar features but with a different wavelength. We therefore assume that the characteristics of the numerical result are the same but redshifted by $\sim 100 \mathrm{~nm}$ compare to the experimental result, and this analysis will provide insight into how a horizontally polarized electric field is distributed on the metamolecule.

The electric field is longitudinally polarized, concentrating on the right and left sides of the nanocantilever. We mechanically simulated pumping of the metamolecules with a horizontally polarized beam at $1200 \mathrm{~nm}$ on a single metamolecule by applying a longitudinal load on one side of the nanocantilever as shown in the inset of Fig. S 10 (b). The activated vibrational modes from the 
surface integral of the nanocantilever in frequency domain are shown in the black curve. The experimental result (red dotted curve) from a horizontal pump excitation is superimposed for comparison. Similar to the modes observed when the pump beam was vertically polarized, the modes at $2.33 \mathrm{GHz}, 5.33 \mathrm{GHz}, 7 \mathrm{GHz}$ and $12 \mathrm{GHz}$ are excited by the longitudinal load. In addition, the excitation of the mode at $3.6 \mathrm{GHz}$ appears to be stronger by the off-center longitudinal load in the simulation results. A weak $3.6 \mathrm{GHz}$ component is also apparent in the experimental result from the horizontally polarized pump beam in Figure $4 \mathrm{c}$ of the main text. The other observed modes from the single metamolecule are not experimentally discernible. In general, while some differences were observed between the dominant mechanical modes of the MSA structure in vertical polarization and horizontal polarization, many of the same modes were excited in both polarizations, which agrees with our experimental observations.

(a)

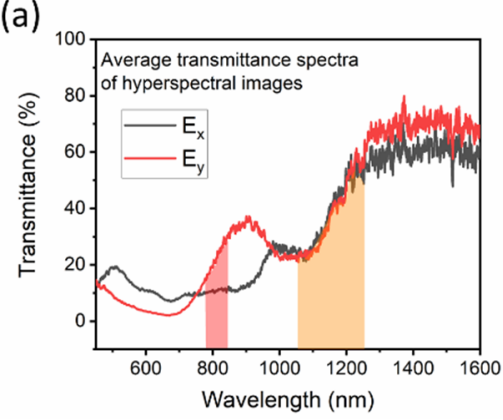

(c)

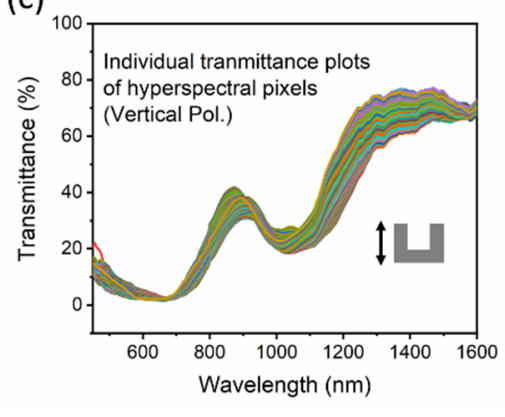

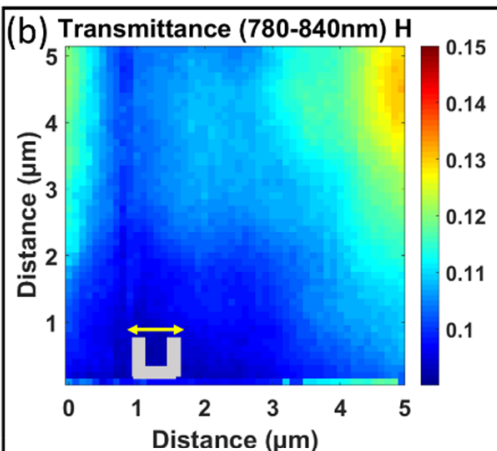

Distance $(\mu \mathrm{m})$

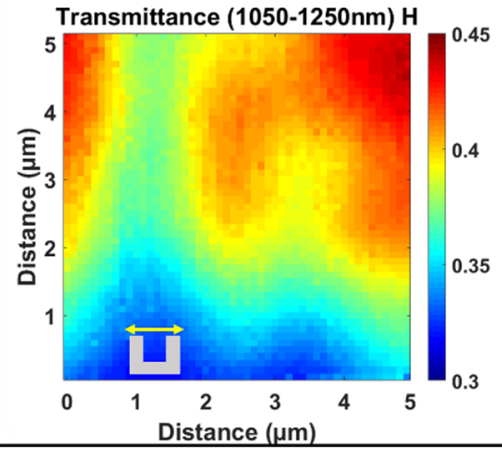

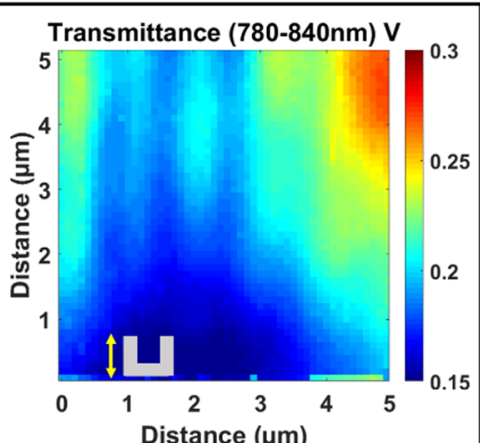

Transmittance $(1050-1250 \mathrm{~nm}) \mathrm{V}$

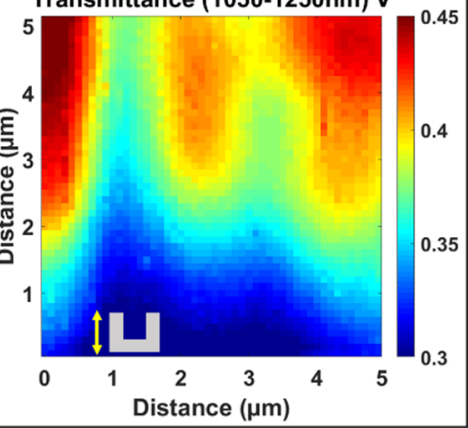

Fig. S 9 (a) Average transmittance spectra from the hyperspectral polarization dependence experiment. (b) Transmission hyperspectral images showing the horizontal and vertical polarization responses, respectively, of a $5 \mu \mathrm{m} \times 5 \mu \mathrm{m}$ area on the MSA at the probe wavelength range (top panels) and pump wavelength range (bottom panels). (c) Transmittance spectra of hyperspectral image pixels for the vertically polarized beam. 
(a)

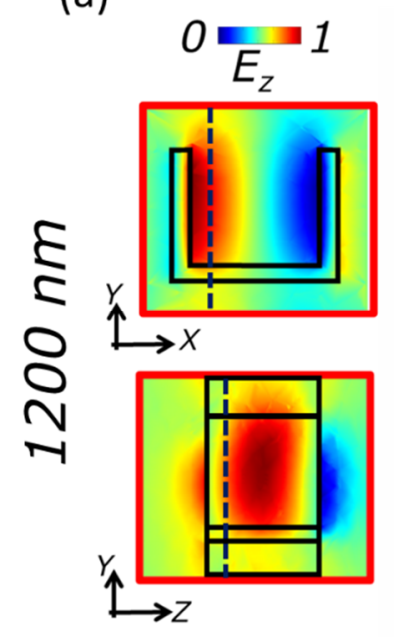

(b)

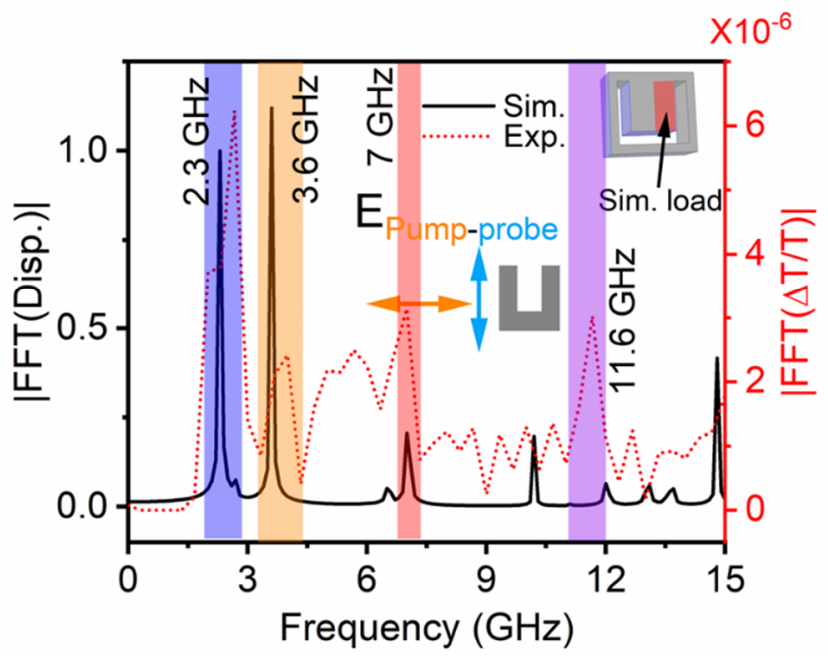

Fig. S 10 (a) Simulated normalized electric field intensity distribution in the $x y$ and zy directions of the metamolecule at $1200 \mathrm{~nm}$. (b) Simulated frequency domain plot of a single metamolecule, showing the normalized mechanical displacement response of the cantilever (solid black) when load is longitudinally applied to the cantilever. The experimental $|\mathrm{FFT}(\Delta \mathrm{T} / \mathrm{T})|$ (dotted red plot)of a horizontal pump and vertical probe beam is superimposed for reference.

To confirm the polarization dependent results discussed, a retake of the pump polarization dependent measurements was carried out at a different location on the MSA. The result, in Fig. S 11 (a), shows that the modes at $\sim 2.33 \mathrm{GHz}$ and $\sim 11 \mathrm{GHz}$ are excited both for vertically polarized pump $\left(\mathrm{E}_{\mathrm{y}-\mathrm{y}}\right)$ and horizontally polarized pumps $\left(E_{x-y}\right)$. This is consistent with the polarization dependent discussion in the manuscript. However, the mode at $\sim 5.33 \mathrm{GHz}$ is excited for $E_{y-y}$ and suppressed for $E_{x-y}$. This is the opposite of what is observed the previous measurements of Figure 4 (c) in the manuscript. This suggests that the $\sim 5.33 \mathrm{GHz}$ mode is not necessarily a polarization induced response. It may be a response due to the slight shift in beam location, while adjusting the polarization optics during the experiment. 
(a)

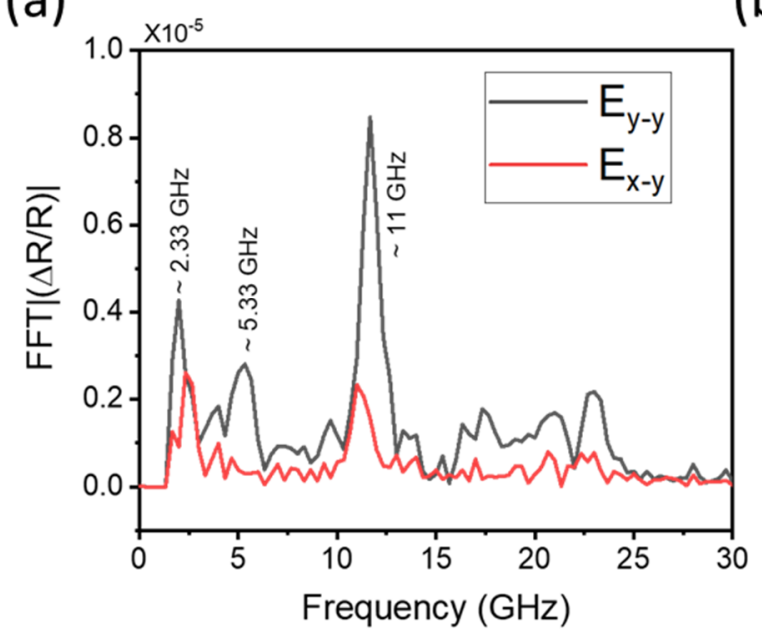

(b)

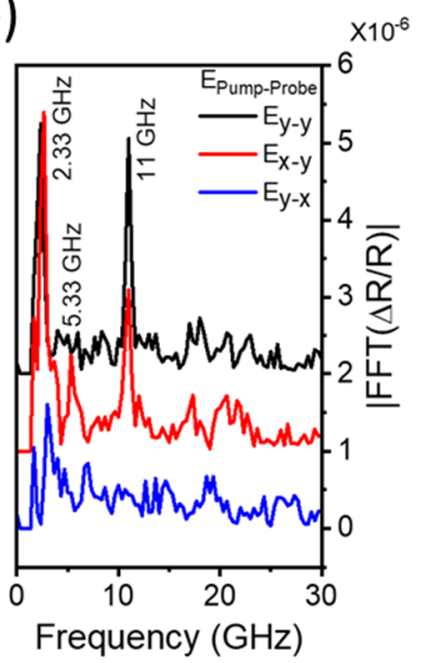

Fig. S 11(a) Frequency-domain plots of the optomechanical responses from a retake of pump polarization dependent measurements at a different location on the metasurface array. (b) Frequency-domain plots of the optomechanical responses of the nanocantilever as represented in Fig. 4 (c) of the manuscript.

\section{S8. Optical force estimation and efficiency}

We use the simulated wavelength dependence of the time averaged optical force to estimate the peak value of the forces, $F_{y}$ and $F_{z}$, acting on the nanocantilever at a pump wavelength of $1100 \mathrm{~nm}$. The pump beam pulse power was measured to be $\sim 24 \mathrm{~W} \mu \mathrm{m}^{-2}$. As shown in Fig. S 12, the normalized forces, Fy and $\mathrm{Fz}$, at $1100 \mathrm{~nm}$, are $0.24 \mathrm{P} / \mathrm{c}$ and $0.37 \mathrm{P} / \mathrm{c}$, respectively, where $\mathrm{P}$ is the input power, and $\mathrm{c}$ is the speed of light. This gives absolute values of $\sim 20 \mathrm{nN} \mu \mathrm{m}^{-2}$ and $\sim 30 \mathrm{nN} \mu \mathrm{m}^{-2}$, for $F_{\mathrm{y}}$ and $F_{z}$, acting on the metamolecule, respectively. The peak at $1050 \mathrm{~nm}$ corresponds to a normalized force of $0.8 \mathrm{P} / \mathrm{c}$ or absolute value of $\sim 65 \mathrm{nN} \mu \mathrm{m}^{-2}$.

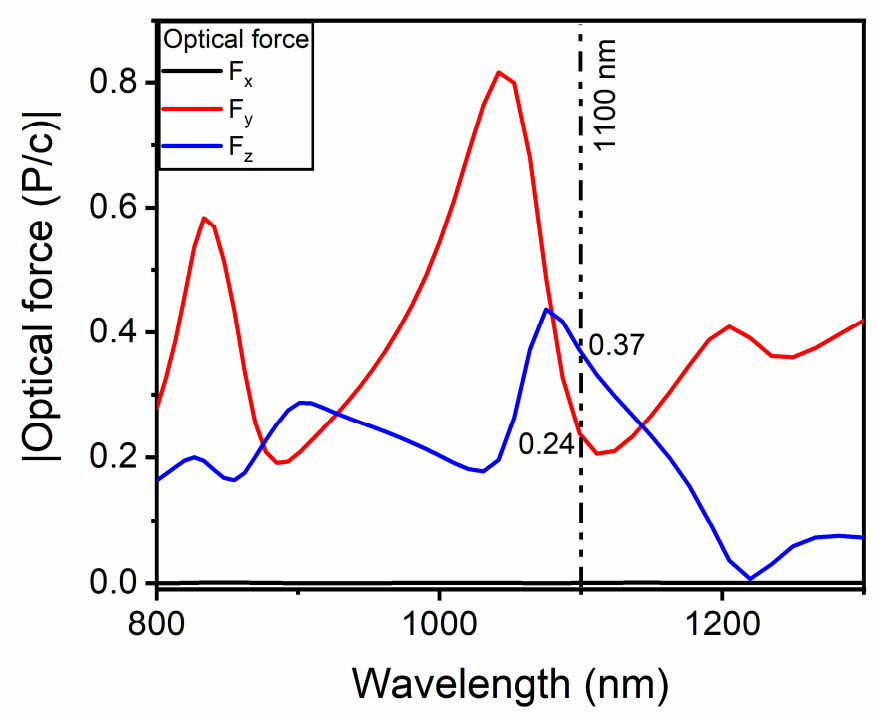

Fig. S 12 Wavelength dependence of the moduli of the optical force magnitudes acting on the nanocantilever in xyz directions. 
Comparing with typical efficiencies of acoustic vibrations obtained using hot electron plasma excitation in nanoparticles also result in displacements in the range of tens of picometers.

Calculating typical strain energies for a typical $L=150 \mathrm{~nm}, A=40 \times 80 \mathrm{~nm}^{2}$ gold nanorod with deformation $\Delta \mathrm{L}=10-100 \mathrm{pm}$ yields $\mathrm{U}=\mathrm{Y} A \Delta \mathrm{L} 2 / 2 \mathrm{~L}=10^{-19}-10^{-17} \mathrm{~J}$, using Youngs modulus $\mathrm{Y}=79 \mathrm{Gpa}$. The efficiency for such picometer vibrational excitations is therefore in the range $10^{-8}-10^{-6}$ of the input energy.

\section{S9. Multi-exponential treatment of fast relaxation process}

While carrier relaxation in the hundreds of ps range has been reported for bulk $\mathrm{SiC}^{9}$, much faster relaxation mechanisms in nanostructured semiconductors can be attributed to the increased effects of surface recombination and fabrication-induced defects on the carrier lifetime. Below-bandgap optical excitation furthermore predominantly results in excitation of short-lived defect states. We have not considered this fast carrier-mediated response in our analysis, as the mechanical response of the MSA takes place over a much longer range. The full measurement range of the experiment is shown in Fig. S 13 (a). To remove the fast carrier effect from our analysis, we apply a tri-exponential model to the signal, as shown in equation 2.

$y(t)=y_{0}+A_{1} e^{-t / \tau_{1}}+A_{2} e^{-t / \tau_{2}}+A_{3} e^{-t / \tau_{3}}$

$A_{1}, A_{2}$ and $A_{3}$ are the maximum amplitudes of the exponential components, $\tau_{1}, \tau_{2}$ and $\tau_{3}$ the decay constants, and $t$ is the independent time variable.

Next, the signal is truncated just after the peak at $\mathrm{t}=0 \mathrm{ps}$, as shown in Fig. $\mathrm{S} 13$ (b). The resulting signal reveals the mechanical response of the metamolecules, shown in Fig. S 13 (c). Subsequently, a Savitzky-Golay smoothening filter over 100 adjacent data points (20 ps moving average) is applied, to reveal the optomechanical oscillations as shown in Fig. $S 13$ (d). 

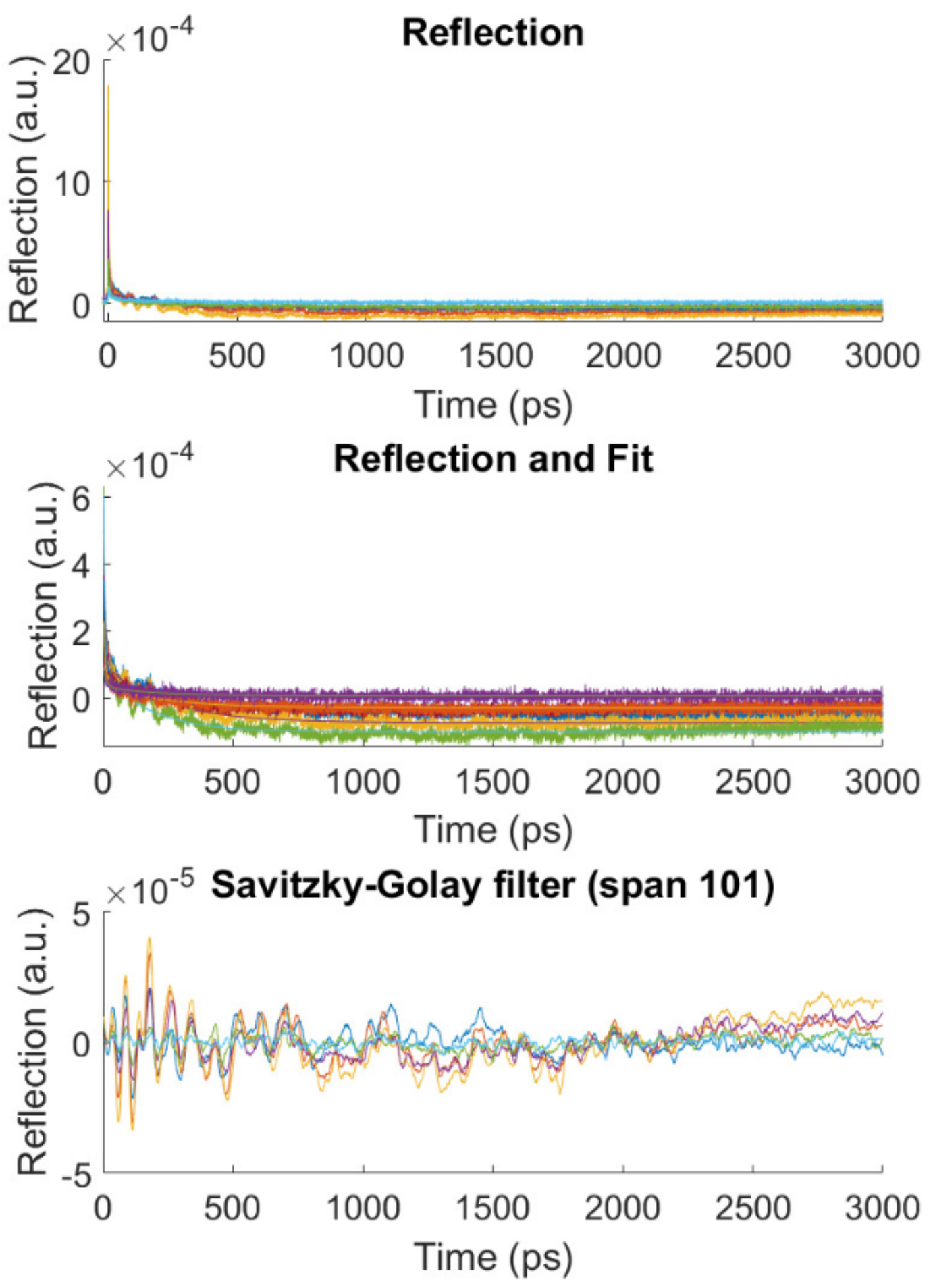

Fig. S 13: Steps in the processing from full traces to vibrational responses for the $\Delta R / R$ signals of Figure 4a. (Top) Reflection signal as measured. (Middle) Signal truncated at zero delay time, showing the experimental response with fitted multi-exponential decay curves. (Bottom) Signal after subtracting fits using the tri-exponential model from Eq. (2), including a 101-point Savitzky-Golay smoothing to reveal the vibrational response. 


\section{References}

1. Ge, S.; Liu, X.; Qiao, X.; Wang, Q.; Xu, Z.; Qiu, J.; Tan, P.-H.; Zhao, J.; Sun, D., Coherent Longitudinal Acoustic Phonon Approaching THz Frequency in Multilayer Molybdenum Disulphide. Sci. Rep. 2014, 4 (1), 5722.

2. $\quad$ Ulbricht, R.; Sakuma, H.; Imade, Y.; Otsuka, P. H.; Tomoda, M.; Matsuda, O.; Kim, H.; Park, G.-W.; Wright, O. B., Elucidating gigahertz acoustic modulation of extraordinary optical transmission through a two-dimensional array of nano-holes. Appl. Phys. Lett. 2017, 110 (9), 091910. 3. Hähnlein, B.; Kovac Jr, J.; Pezoldt, J., Size effect of the silicon carbide Young's modulus. physica status solidi (a) 2017, 214 (4), 1600390.

4. Harris, G. L.; INSPEC, Properties of Silicon Carbide. INSPEC, Institution of Electrical Engineers: 1995.

5. Wang, S.; Zhan, M.; Wang, G.; Xuan, H.; Zhang, W.; Liu, C.; Xu, C.; Liu, Y.; Wei, Z.; Chen, X., 4H-SiC: a new nonlinear material for midinfrared lasers. Laser \& Photonics Reviews 2013, 7 (5), 831-838.

6. Henins, I., Precision Density Measurement of Silicon. Journal of research of the National Bureau of Standards. Section A, Physics and chemistry 1964, 68A (5), 529-533.

7. Boyd, E. J.; Uttamchandani, D., Measurement of the Anisotropy of Young's Modulus in Single-Crystal Silicon. JMemS 2012, 21 (1), 243-249.

8. Aspnes, D. E.; Studna, A. A., Dielectric functions and optical parameters of Si, Ge, GaP, GaAs, GaSb, InP, InAs, and InSb from 1.5 to 6.0 eV. Phys. Rev. B 1983, 27 (2), 985-1009.

9. Fang, Y.; Nie, Y.; Wu, X.; Yang, J.; Chen, Y.; Wang, Y.; Wu, Q.; Song, Y., Excitation wavelength-dependent carrier dynamics in $\mathrm{n}$-type and semi-insulating $6 \mathrm{H}-\mathrm{SiC}$ using ultrafast transient absorption spectroscopy. J. Appl. Phys. 2019, 125 (23), 235703. 